\title{
RESULTATS DU TRAITEMENT CHIRURGICAL DE L'OTOSPONGIOSE NOTRE EXPERIENCE A PROPOS DE 310 CAS
}

\author{
R. MANI, T. BEN MAKHLOUF, W. KERMANI, M. BELLAKHDHAR, I. HARRABI ${ }^{*}$, I. ZEGLAOUI, \\ M. BEN ALI, M. ABDELKÉFI, M. BELCADHI, K. BOUZOUITA. \\ SERVICE D'ORL ET DE CHIRURGIE CERVICO-FACIALE \\ * SERVICE D'ÉPIDÉMIOLOGIE \\ HÔPITAL UNIVERSITAIRE FARHAT HACHED SOUSSE
}

\begin{abstract}
Introduction : Le traitement de l'otospongiose est chirurgical. Plusieurs facteurs peuvent intervenir dans l'indication opératoire ou influencer les résultats fonctionnels. L'objectif de notre étude est d'évaluer nos résultats audiométriques et d'étudier les facteurs prédictifs de l'échec fonctionnel.

Matériel et méthodes : II s'agit d'une étude rétrospective à propos de 310 oreilles opérées pour otospongiose, sur une période de 21 ans (1985-2005). On a effectué une analyse des résultats audiométriques à partir des audiogrammes postopératoires faits à 3 mois, à 1 an et au dernier contrôle. Nous avons ensuite étudié 8 facteurs pouvant être prédictifs de l'échec fonctionnel. Ce dernier était défini par un Rinne résiduel post-opératoire (RRPO) > $10 \mathrm{~dB}$.

Résultats : Le RRPO était en moyenne de $7,9 \mathrm{~dB}$ avec des extrêmes de $5 \mathrm{~dB}$ à $33 \mathrm{~dB}$. La fermeture du Rinne a été obtenue dans $87,3 \%$ des cas.

Après étude multivariée avec régression logistique, nous avons retenu 2 facteurs indépendants significativement prédictifs de l'échec fonctionnel : un stade audiométrique III ou IV d'Aubry (OR : 19,06/p : 0,0001) et un Rinne post-opératoire précoce supérieur à $35 \mathrm{~dB}$ (OR : $4,01 / \mathrm{p}: 0,0025)$.

Conclusion : Une audiométrie post-opératoire précoce serait intéressante pour dépister précocement un échec chirurgical d'une part et pour préjuger du résultat fonctionnel définitif d'autre part. Un stade audiométrique III ou IV d'Aubry, prédictif certes d'un moins bon résultat fonctionnel, ne contre-indique pas la chirurgie qui permettra entre autres une meilleure adaptation audio- prothétique.
\end{abstract}

Mots-clés : otospongiose - chirurgie - résultats - facteurs prédictifs

\begin{abstract}
SUMMARY
Introduction : Surgery is the treatment of choice for otosclerosis. The aim of this study was to evaluate the results of stapes surgery and to investigate predictive factors in the postoperative hearing outcomes.

Materials and methods : A retrospective study was conducted from January 1985 to December 2005. During this period, 310 stapedectomies for otosclerosis were performed in our department. Post-operative air conduction and bone conduction thresholds at 3 months, 1 years and at the last control were studied. Eight factors that may predict functional failure in the post-operative hearing outcomes have also been studied. The criterion for failure was defined as closure of the air-bone gap greater than $10 \mathrm{~dB}$. Statistical analysis was carried out using logistic regression.
\end{abstract}

Results : The mean post-operative air-bone gap was 7,9 dB. Air-bone gap closure within $10 \mathrm{~dB}$ was obtained in 87,3 percent of cases. Using regression model, the factors found to be significantly correlated with a post-operative air-bone gap greater than $10 \mathrm{~dB}$ are advanced audiometric stage (III, IV) (OR: 19,06/ p: 0,0001) and early post-operative air-bone gap>35 dB ( OR: 4,01/p: 0,0025)

Conclusion : Early post-operative air-bone gap is useful to detect early failure of stapes surgery. Although advanced otosclerosis was considered a negative factor for hearing gain, thanks to stapes surgery patients with advanced otosclerosis can better benefit from appropriate hearing aids.

Keywords : otosclerosis - surgery - results - predictive factors

\section{INTRODUCTION}

L'otospongiose est une ostéodystrophie primitive de la capsule otique, responsable lorsqu'elle se manifeste cliniquement, d'une surdité de transmission (ou d'une surdité mixte à prédominance transmissionnelle) par ankylose stapédo-vestibulaire. Son traitement repose essentiellement sur la chirurgie.

Le choix de la technique opératoire a été largement étudié dans la littérature comme facteur prédictif de la qualité du résultat fonctionnel de la chirurgie. D'autres facteurs 
tels que l'âge, la durée d'évolution, le stade audiométrique, pouvant intervenir dans l'indication opératoire ou influencer ces résultats fonctionnels ont été peu évalués. Le but de notre étude était :

d'évaluer les résultats audiométriques des oreilles opérées.

d'étudier les facteurs prédictifs de l'échec fonctionnel, dans le but d'une sélection plus fine des indications opératoires et d'un choix plus approprié de la technique chirurgicale.

\section{MATÉRIEL ET MÉTHODES}

De Janvier 1985 à Décembre 2005 (21 ans), 252 dossiers de malades opérés pour otospongiose au service d'ORL et de chirurgie cervico-faciale du CHU Farhat Hached de Sousse, ont été colligés. Parmi eux, 58 patients ont été opérés des deux oreilles, ce qui porte le nombre d'oreilles opérées à 310 . Un suivi minimal de 3 mois après le traitement a été exigé, avec au moins un contrôle audiométrique post-opératoire.

L'étude a comporté deux parties:

- Une analyse des résultats audiométriques à partir des audiogrammes post-opératoires faits à 3 mois, 1 an et au dernier contrôle. Les résultats ont été exprimés en calculant le Rinne résiduel post opératoire (RRPO) : différence entre la moyenne des seuils post-opératoires en conduction aérienne (CA) et conduction osseuse (CO) sur les fréquences 500,1000 et $2000 \mathrm{~Hz}$. Un succès chirurgical était défini par un RRPO inférieur ou égal à 10 décibels, et en évaluant la réserve cochléaire (ERC) : différence entre les seuils moyens en CO sur les fréquences 1000, 2000 et $4000 \mathrm{~Hz}$ en pré et post-opératoire. Une valeur négative de la réserve cochléaire correspond à une amélioration de la $\mathrm{CO}$, une valeur positive correspond à une baisse de la $\mathrm{CO}$ ou labyrinthisation.

- Une étude des facteurs prédictifs de l'échec fonctionnel : 8 variables ont été étudiées :

1 -Age

2-Sexe

3-Stade audiométrique d'Aubry

4-Variation anatomique du canal de Fallope

5-Geste platinaire

6-Matériel d'interposition

7-Persistance d'un vertige post-opératoire (au-delà du troisième jour)

8-Rinne post-opératoire précoce (CA précoce moyenne CO précoce moyenne): calculé à partir d'un audiogramme post-opératoire précoce fait à $\mathrm{J} 6, \mathrm{~J} 7 \mathrm{ou} \mathrm{J} 8$ post opératoire, avec calcul de la moyenne sur les fréquences 500 , 1000, $2000 \mathrm{~Hz}$.

Un échec fonctionnel était défini par un RRPO > $10 \mathrm{~dB}$ calculé à 3 mois en post-opératoire.

La saisie et le traitement des données ont été réalisés à l'aide du logiciel SPSS (version 9.0). Nous avons procédé dans une première étape à une analyse univariée à la recherche d'une corrélation entre la variable dépendante (échec fonctionnel) et les variables explicatives prises une par une. Notre population a été répartie en deux groupes (tableau I).

\begin{tabular}{|l|l|}
\hline Variable à l'étude & Unité \\
\hline Age (an) & $<35$ / $>35$ \\
\hline Sexe & Masculin / Féminin \\
\hline Stade audiométrique d'Aubry & Stade I - II /stade III - IV \\
\hline Variation anatomique du canal de Fallope & Oui / non \\
\hline Geste platinaire & Platinectomie totale / platinotomie \\
\hline Matériel d'interposition & Veine / aponévrose \\
\hline Rinne post-opératoire précoce(dB) & $<35 \mathrm{~dB} />35 \mathrm{~dB}$ \\
\hline Persistance d'un vertige post-opératoire & Oui / non \\
\hline
\end{tabular}

Tableau I : Unités de séparation des variables de l'étude des facteurs prédictifs de l'échec fonctionnel

Pour les variables quantitatives (âge, RPOP) l'unité de séparation correspond à la médiane des valeurs obtenues.

La validation des résultats obtenus a été réalisée grâce à un test Chi-deux pour les variables qualitatives et le test $\mathrm{T}$ de Student pour les variables quantitatives.

Dans une deuxième étape, nous avons procédé à une étude multivariée. Cette étape permet de sélectionner les variables retenues comme étant à relation significative avec l'échec fonctionnel lors de l'étude univariée et de les soumettre à une régression logistique afin d'éliminer toute interdépendance entre les différentes variables, étape nécessaire pour valider l'étude et sans laquelle notre étude serait biaisée. Pour toutes nos analyses statistiques, le seuil de signification a été fixé à $5 \%$.

\section{RÉSULTATS}

L'âge moyen de nos malades au moment de la chirurgie était de 35,8 ans (des extrêmes de 14 et 63 ans). Les malades étaient âgés de 20 à 40 ans dans $68,3 \%$ des cas. L'otospongiose juvénile (celle survenant avant 20 ans) avait représenté $3,9 \%$ de nos otospongioses opérées. Une prédominance féminine était notée avec 178 $(70,6 \%)$ malades de sexe féminin contre $74(29,4 \%)$ malades de sexe masculin. Le sex-ratio était de 2,4 pour les femmes.

Selon la classification audiométrique d'Aubry, 18 malades $(5,2 \%)$ étaient à un stade I, 237 malades $(76,5 \%)$ au stade II, 53 malades $(17,1 \%)$ au stade III et 2 malades $(0,6 \%)$ au stade IV.

Le Rinne pré-opératoire moyen variait de $15 \mathrm{~dB}$ à $54 \mathrm{~dB}$ avec une moyenne de $31,5 \mathrm{~dB} .53,9 \%$ de nos patients avaient un Rinne pré-opératoire moyen $>30 \mathrm{~dB}$. 
Tous nos malades ont été opérés sous anesthésie générale. Cinquante huit malades ont été opérés des deux cotés, ce qui porte le nombre total d'oreilles opérées à 310. L'oreille opérée était droite dans 161 cas et gauche dans 149 cas.

Cinquante et une oreilles ont été opérées par voie intraméatique, 259 oreilles par voie endaurale de Shambaugh. Le geste platinaire était : -une platinectomie totale dans 160 cas $(51,6 \%)$.

-une platinectomie partielle dans 101 cas $(32,6 \%)$.

-une platinotomie dans 49 cas (15,8\%).

Le matériel d'interposition était une aponévrose dans 164 cas $(61,2 \%)$ et une veine dans 104 cas $(38,8 \%)$ (40 oreilles opérées d'une platinotomie calibrée sans interposition ont été exclues ainsi que deux oreilles Geyser).

La prothèse utilisée était un piston en téflon de $0,4 \mathrm{~mm}$ de calibre dont la longueur était adaptée à la distance entre la branche descendante de l'enclume et la fenêtre ovale. Une anomalie anatomique du canal de Fallope (procidence et/ou déhiscence) a été retrouvée dans 66 cas $(21,3 \%)$, à type de :

- Procidence : 40 malades $(60,6 \%)$.

- Déhiscence : 17 malades (25,8\%).

- Procidence et déhiscence : 9 malades (13,6\%).

Une antibiothérapie prophylactique, ainsi qu'un traitement vasodilatateur, corticoïde et anti-vertigineux ont été instaurés de façon systématique chez nos malades. Un antiémétique a été prescrit pour 135 malades (43,5\%).

Un vertige post-opératoire persistant a été signalé par 68 malades (22\%). Une infection post-opératoire a été constatée chez 10 malades (3,2\%).

Le recul moyen de nos malades était de 22 mois avec un minimum de 3 mois, un maximum de 17 ans. Quatre oreilles ont été exclues de l'étude audiométrique: 2 reprises pour échec fonctionnel avant 3 mois et 2 oreilles Geyser.

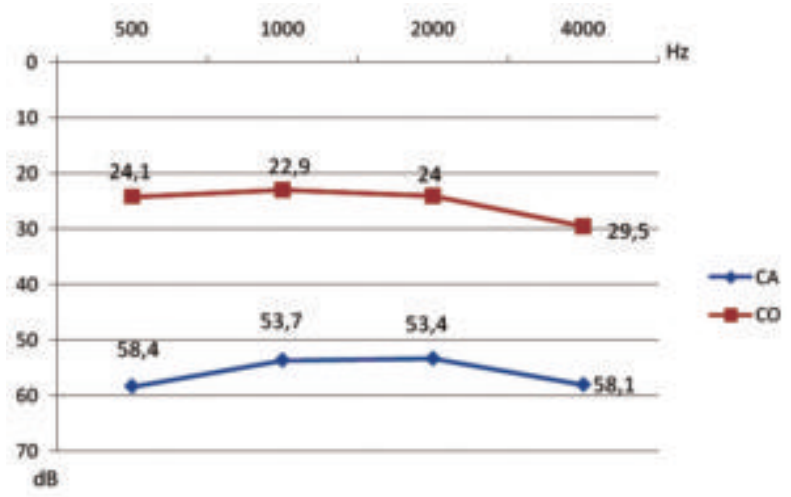

Fig. 1 : Audiogramme pré-opératoire moyen

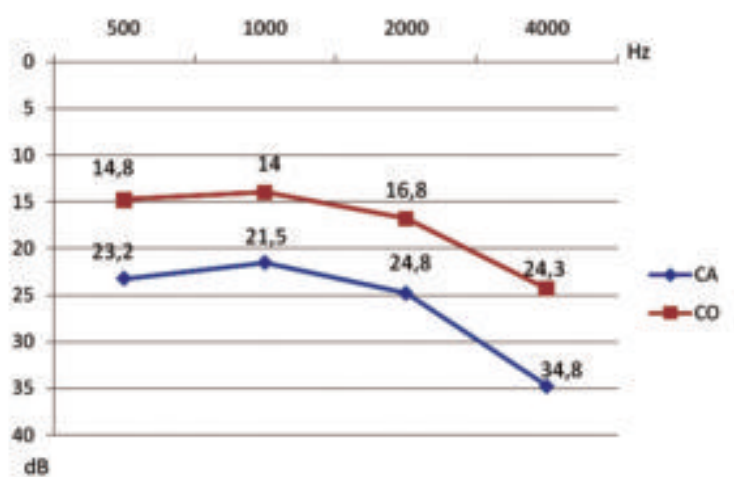

Fig. 2 : Audiogramme post-opératoire moyen au dernier controle

A 3 mois, le RRPO était en moyenne de 7,9 dB avec des extrêmes de $5 \mathrm{~dB}$ à $33 \mathrm{~dB}$ (figure 1,2). On a constaté :

- La fermeture du Rinne (RRPO $\leq 10 \mathrm{~dB})$ a été obtenue dans $87,3 \%$ des cas.

$-10<$ RRPO $\leq 20 \mathrm{~dB}$ dans $10 \%$ des cas.

- RRPO > $20 \mathrm{~dB}$ dans $2,7 \%$ des cas.

$A$ un an, le RRPO était en moyenne de $8,1 \mathrm{~dB}$ avec des extrêmes de $5 \mathrm{~dB}$ et $28 \mathrm{~dB}$. La fermeture du Rinne a été obtenue dans $81,1 \%$ des cas.

Au dernier contrôle, le RRPO était en moyenne de 8,3 dB avec des extrêmes de $5 \mathrm{~dB}$ à $35 \mathrm{~dB}$. La fermeture du Rinne a été obtenue dans $80,9 \%$ des cas.

L'étude de la réserve cochléaire a montré :

- A trois mois, une amélioration de la réserve cochléaire dans $78,8 \%$ des cas. Elle est demeurée inchangée dans $8,5 \%$ des cas, et s'est dégradée dans $12,7 \%$ des cas. Le taux d'amélioration était meilleur de façon non significative $(p=0,641)$ après platinotomie $(86,4 \%)$ qu'après platinectomie totale $(83,4 \%)$.

- A un an, une amélioration de la réserve cochléaire dans $80,5 \%$ des cas.

- Au dernier contrôle, une amélioration de la réserve cochléaire dans $77,5 \%$ des cas.

Cinq malades ont présenté une cophose post-opératoire $(1,6 \%), 3$ d'entre elles étaient survenues d'une façon précoce (2 infections post-opératoires, 1 Geyser), les deux autres après un intervalle de 1 an et 4 ans.

\section{- Etude univariée des facteurs de risque de l'échec fonctionnel :}

Lors de l'étude univariée, deux variables ont été retenues comme ayant une relation significative avec l'échec fonctionnel (valeur de $p<5 \%$ ) ; il s'agit du stade audiométrique d'Aubry et du Rinne post-opératoire précoce (tableau II).

-Après étude multivariée avec régression logistique: Nous avons retenu ces 2 mêmes facteurs comme facteurs indépendants significativement prédictifs de l'échec fonctionnel : un stade audiométrique III ou IV d'Aubry (OR : 19,06/p : 0,0001 ) et un RPOP supérieur à $35 \mathrm{~dB}$ (OR : $4,01 / p$ : 0,0025). 


\begin{tabular}{|c|c|c|c|c|}
\hline \multicolumn{2}{|c|}{ Variable à l'étude } & Pourcentage & Odds Ratio & $\mathbf{p}$ \\
\hline \multirow{2}{*}{ Age } & $\leq 35$ & $85,4 \%$ & \multirow{2}{*}{1,38} & \multirow{2}{*}{0,3463} \\
\hline & $>35$ & $89 \%$ & & \\
\hline \multirow{2}{*}{ Sexe } & Masculin & $87,6 \%$ & \multirow{2}{*}{1,05} & \multirow{2}{*}{0,8970} \\
\hline & Féminin & $87,1 \%$ & & \\
\hline \multirow{2}{*}{ Stade audiométrique } & Stade I - II & $94 \%$ & \multirow{2}{*}{12,63} & \multirow{2}{*}{0,0001} \\
\hline & Stade III - IV & $55,6 \%$ & & \\
\hline \multirow{2}{*}{$\begin{array}{l}\text { Variation anatomique } \\
\text { du canal de Fallope }\end{array}$} & Oui & $87,5 \%$ & \multirow{2}{*}{1,03} & \multirow{2}{*}{0,9473} \\
\hline & Non & $87,2 \%$ & & \\
\hline \multirow{2}{*}{ Geste platinaire } & Platinectomie $\mathrm{T}$ & $85,5 \%$ & \multirow{2}{*}{1,01} & \multirow{2}{*}{0,9838} \\
\hline & Platinotomie & $85,4 \%$ & & \\
\hline \multirow{2}{*}{ Matériel d'interposition } & Veine & $86,7 \%$ & \multirow{2}{*}{1,09} & \multirow{2}{*}{0,8131} \\
\hline & Aponévrose & $87,7 \%$ & & \\
\hline \multirow{2}{*}{ Rinne post op précoce } & $\leq 35 \mathrm{~dB}$ & $89,2 \%$ & \multirow{2}{*}{2,12} & \multirow{2}{*}{0,0407} \\
\hline & $>35 \mathrm{~dB}$ & $75 \%$ & & \\
\hline \multirow{2}{*}{$\begin{array}{l}\text { Persistance d'un vertige } \\
\text { post opératoire }\end{array}$} & Oui & $84,8 \%$ & \multirow{2}{*}{1,3} & \multirow{2}{*}{0,5089} \\
\hline & Non & $87,9 \%$ & & \\
\hline
\end{tabular}

Tableau II : Taux de fermeture complète du Rinne selon les facteurs de l'étude

\section{DISCUSSION}

Le traitement de choix de l'otospongiose repose sur la chirurgie. Pour plusieurs auteurs, la perte auditive moyenne à partir de laquelle une intervention est indiquée est d'au moins $30 \mathrm{~dB}(1,2)$. Dans notre série, la moyenne des Rinnes pré-opératoires moyens était de $31,5 \mathrm{~dB}$ avec des extrêmes allants de $15 \mathrm{~dB}$ à $54 \mathrm{~dB} .53,9 \%$ de nos patients avaient un Rinne pré-opératoire moyen supérieur à 30 $\mathrm{dB}$.

Le taux de réussite, défini par la plupart des auteurs par un RRPO $\leq 10 \mathrm{~dB}$ a été obtenu dans notre série lors $\mathrm{du}$ premier contrôle (3 mois) dans $87,3 \%$ des cas. Ce taux est comparable à celui rapporté dans les séries de la littérature avec un premier contrôle allant de 1 à 3 mois (tableau III).

\begin{tabular}{|c|c|c|}
\hline Auteurs & Nombre d'oreilles & Taux de réussite \\
\hline Mangham (4) & 215 & $96 \%$ \\
\hline Herzog (5) & 65 & $87 \%$ \\
\hline Quaranta (6) & 151 & $84,7 \%$ \\
\hline Bourguignat (3) & 67 & $73 \%$ \\
\hline Notre série & 306 & $87,3 \%$ \\
\hline
\end{tabular}

Tableau III : Pourcentage de fermeture du Rinne selon la littérature avec un premier contrôle allant de 1 à 3 mois
Après un recul moyen de 22 mois avec des extrêmes allant de 3 à 204 mois, le pourcentage de fermeture du Rinne au dernier contrôle chez nos malades était de l'ordre de $80,9 \%$. Ce pourcentage est aussi comparable à celui de la littérature.

L'étude de la $\mathrm{CO}$ dans les résultats de l'otospongiose est capital, car elle est le reflet de la réserve cochléaire (7). La remontée de la $\mathrm{CO}$ en post-opératoire sur les fréquences graves est quasi constante. Dubreuil (8) a constaté une amélioration ou une conservation de la CO dans $91 \%$ des cas, Beal (9) a retrouvé $80 \%$ de gain à court terme, Moscillo (10) a obtenu $95 \%$ de gain quelque soit la technique opératoire. Dans notre série, l'amélioration de la CO était observée dans $78,8 \%$ des cas avec un gain moyen de $7,1 \mathrm{~dB}$.

Le début de l'otospongiose est souvent difficile à préciser, c'est plutôt l'âge au moment de l'intervention qui est généralement retenu. L'âge moyen était de 52 ans pour Schea (1). Pour Besbes une prédominance des adultes jeunes a été notée avec dans $61,4 \%$ des cas un âge compris entre 20 et 39 ans (2). Dans notre série, l'âge à l'intervention était compris entre 14 et 63 ans avec une moyenne de 35,8 ans. $68,3 \%$ de nos patients avaient un âge entre 20 et 40 ans

Les formes juvéniles survenant avant l'âge de 20 ans sont rares. Elles ne constituent pour Schea que moins de 2,3\% (1). Dans la série de Besbes, elle représente 9,9\% des malades (2). Dans notre série, elle a été observée dans $3,9 \%$ des cas.

L'effet de l'âge sur les résultats fonctionnels est apprécié de façon diverse selon les auteurs.

Dans notre série, on a obtenu de meilleurs résultats chez les sujets âgés de plus de 35 ans avec une fermeture du Rinne dans $89 \%$ des cas contre $85,4 \%$ des cas chez les sujets âgés de moins de 35 ans, mais cette différence n'était pas significative $(p=0,35)$. Pour Bourgignat, les gains en $\mathrm{CO}$ et en $\mathrm{CA}$ ainsi que le Rinne post-opératoire n'ont pas été corrélés avec l'âge (3). Dans notre série, les résultats audiométriques étaient moins bons en cas d'otospongiose juvénile (fermeture du Rinne dans $81,1 \%$ des cas) comparée à ceux obtenus chez les adultes (fermeture du Rinne dans $87,5 \%$ des cas), avec une différence non significative $(p=0,582)$. Vincent (11), en opérant 28 patients otospongieux âgés de moins de 18 ans, a obtenu une fermeture du Rinne en post-opératoire dans $93,5 \%$ des cas. Millman (12) a obtenu un taux de réussite de $58 \%$ après stapédectomie. Romanet, en opérant 8 cas d'otospongiose juvénile (avant 15 ans) a obtenu une fermeture du Rinne dans 63\% des cas (13). II a conclu que ces résultats n'étaient pas mauvais, mais ils restaient néanmoins inférieurs à ceux obtenus chez l'adulte.

Une prédominance féminine de l'otospongiose clinique a été retrouvée dans la plupart des séries $(8,14,15)$. Par contre, dans l'otospongiose histologique, la différence entre les deux sexes est non significative $(16,17)$. Dans 
notre série, une prédominance féminine a été retrouvée avec 178 femmes $(70,6 \%)$ pour 74 hommes $(29,4 \%)$, le sex-ratio était de 2,4 pour les femmes. On n'a pas trouvé d'influence significative du sexe sur la qualité des résultats fonctionnels obtenus. Le taux de fermeture complète du Rinne était de $87,6 \%$ chez les patients de sexe masculin et de $87,1 \%$ pour les patients de sexe féminin. Vartiainen (18) n'a pas noté lui aussi de corrélation entre la fermeture du Rinne et le sexe.

Les résultats audiométriques sont variables en fonction du stade évolutif de la maladie otospongieuse. Dans notre série, un stade III ou IV d'Aubry a été retenu comme facteur significativement prédictif d'échec fonctionnel (Odds Ratio $=19,06 / p=0,0001)$. Pour Besbes (2), le taux de fermeture complète du Rinne passe de $47,4 \%$ au stade I d'Aubry, à 27,5 \% au stade IV d'Aubry. Causse (19), en opérant des malades au stade sub-cophotique, a pu constater que les résultats sont excellents à condition de n'entraîner lors de l'intervention aucun mouvement excessif des liquides labyrinthiques, de n'envisager l'intervention que lorsqu'il existe un weber osseux encore latéralisé vers l'oreille la plus sourde, et de prescrire un traitement médical vasculaire et enzymatique. Satar (20) a obtenu un pourcentage de fermeture du Rinne meilleur chez les patients avec une bonne réserve cochléaire mais sans être significatif (4\% Vs 29\%) alors que l'amélioration de la courbe osseuse était statistiquement meilleure chez ces mêmes malades $(p<0,001)$. Khalifa (17) en opérant 8 malades otospongieux au stade sub-cophotique a pu restaurer un niveau d'audition permettant une adaptation prothétique conventionnelle dans 6 cas. Zaki (21) a rapporté une série de 9 oreilles non appareillables opérées pour surdité mixte profonde par otospongiose avec un taux de succès dans 8 cas.

Le choix de la voie d'abord dépend des habitudes du chirurgien et des conditions anatomiques locales $(22,23)$. Dans notre série la voie d'abord était une voie endaurale de Shambaugh dans $83,5 \%$ des cas, et une voie intraméatique dans $16,5 \%$ des cas. Nous n'avons pas retrouvé de différence significative des résultats audiométriques entre ces deux voies.

Dans notre série, nous n'avons pas trouvé de différence significative des résultats audiométriques entre les platinectomies totales (taux de fermeture complète du Rinne $=85,5 \%$ ) et les platinotomies (taux de fermeture complète du Rinne $=85,4 \%$ ). Rondini-Gilli (24) et Esquivel (25) n'ont pas constaté de différence statistiquement significative entre platinectomie et platinotomie pour le Rinne résiduel sur les fréquences moyennes.

La nature du greffon utilisé dépend également des habitudes du chirurgien et de la voie d'abord effectuée $(22,23)$. Dans notre série, le matériel d'interposition était une aponévrose dans $61,2 \%$ des cas et une veine dans $38,8 \%$ des cas. On n'a pas trouvé de différence significative dans les résultats audiométriques entre l'interposition de veine (fermeture du Rinne dans $86,7 \%$ des cas) et l'interposition d'aponévrose temporale (fermeture du Rinne dans $87,6 \%$ des cas).

L'incidence des anomalies anatomiques du canal de Fallope varie dans la littérature selon que l'exposition et l'examen du canal facial soient réalisés de façon systématique ou non et selon qu'on prenne ou non en compte les simples déhiscence du canal de Fallope $(26,27)$. L'existence de variations anatomiques du canal de Fallope n'a pas influencé la qualité de nos résultats audiométriques. Dans ces cas, le taux de fermeture complète du Rinne était de $87,5 \%$. Daniels (28) n'a pas noté lui aussi une influence significative des variations anatomiques du canal de Fallope sur les résultats audiométriques.

La fréquence de survenue de Geyser labyrinthique varie selon la littérature de $0,003 \%$ à $0,5 \%(15,29)$. Dans notre série, cet incident est survenu dans 2 cas $(0,6 \%)$. L'attitude chirurgicale avait consisté en un colmatage du trou de sécurité platinaire avec interruption de l'intervention.

Les vertiges sont fréquemment signalés les premiers jours post-opératoires. Dubreuil (8) a constaté des vertiges invalidants dans $2,2 \%$ des cas et Simoncelli dans $1 \%$ des cas (15). Dans notre étude, une fermeture du Rinne était meilleure en absence de d'un vertige postopératoire persistant $(87,9 \%$ contre $84,8 \%)$, mais cette différence n'était pas significative $(p=0,051)$.

Enfin, nous pratiquons dans notre service de façon systématique aux alentours du septième jour post-opératoire un audiogramme post-opératoire précoce. II a comme but de révéler précocement un échec fonctionnel qui, avec le reste du contexte clinique, pourrait indiquer une reprise chirurgicale précoce. Dans notre étude un RPOP $>35 \mathrm{~dB}$ a été retenu comme facteur significativement prédictif d'échec fonctionnel $(O R=4,01 / p=0,0025)$. La fermeture du Rinne a été obtenue dans $89,2 \%$ des cas en présence d'un RPOP $\leq 35 \mathrm{~dB}$ contre $75 \%$ des cas en présence d'un RPOP > $35 \mathrm{~dB}$.

\section{CONCLUSION}

De notre étude émergent 2 facteurs indépendants significativement prédictifs de l'échec fonctionnel: un RPOP supérieur à $35 \mathrm{~dB}$ et un stade audiométrique III ou IV d'Aubry.

Une audiométrie post-opératoire précoce serait donc intéressante pour dépister précocement un échec chirurgical d'une part et pour préjuger du résultat fonctionnel définitif d'autre part.

Concernant le stade audiométrique pré-opératoire, le pourcentage de fermeture du Rinne moins bon en cas d'otospongiose évoluée (stade audiométrique III ou IV) ne contre-indique pas la chirurgie qui reste toujours bénéfique pour permettre entre autres, une meilleure adaptation audio- prothétique. 


\section{REFERENCES}

1-Shea JJ. Forty years of stapes surgery. Am J Otol 1998; 19: 52-5. 2-Besbes G, Akrout A, Belcadhi M, et al. L’otospongiose : données épidémiologiques, résultats du traitement. Rev Laryngol Otol Rhinol 1994 ; 115 : 49-52. 3-Bourguignat E, Roulleau P. Otospongiose : étude de l'évolution de l'audition post-opératoire dans les cinq premières années et recherche de certains facteurs pronostiques. A propos d'une série de 67 cas. Ann Otolaryngol Chir CErvicofac $1994 ; 111: 3-21$.

4-Mangham CA, Reducing footplate complications in small fenestra microdrill stapedotomy. Am J Otol 1993; 14: 118-21.

5-Herzog JA. 0,4 mm stapedotomy: a consistent technique for otosclerosis. Am J Otol 1991; 12: 16-9.

6-Guaranta N, Besossi G, Fallacara RA, Quaranta A. Air and bone conduction change after stapedotomy and partial stapedectomy for otosclerosis. Otolaryngol Head Neck Surg 2005; 133: 116-20.

7-Dubreuil C, Nouchayer M, Boulud B, Di Brango P, Reiss T. Otospongiose : platinotomie ou platinectomie. Etude comparative à long terme. A propos de 1279 cas. Ann Otolaryngol Chir Cervicofac 1994 ; 111 : 249-64.

8-Bouzouita J, Amari F, Gassab I, Sayadi SJ, Gassab A. Platinotomie versus platinectomie dans le traitement de l'otospongiose : 94 interventions. J Tun ORL $2001 ; 7: 21-4$

9-Beal C, Poncet-Wallet C, Frachet B, Ouayoun M. A propos de l'effet Carhart, étude de l'évolution post- opératoire de 47 otospongioses opérées. Ann Otolaryngol Chir Cervicofac $1992 ; 109: 76-9$

10-Moscillo L, Imperiali M, Carra P, Catapano F, Motta G. Bone conduction variation poststapedotomy. Am J Otol 2006; 27: 330-3.

11- Vincent R, Sperling NM, Oates J, Jindal M. Surgical findings and long-term hearing results in 3050 stapedotomies for primary otosclerosis: a prospective study with the otology-nneurology database. Otol Neurotol 2006; 27: 25-47. 12-Millman B, Giddings NA, Cole JM. Long-term follow-up of stapedectomy in children and adolescents. Otolaryngol Head Neck Surg 1996; 115: 78-81.

13-Romanet $P$, Morizot $B$,Creuzot $J$. L'otospongiose juvenile, réflexions à propos d'une statistique personnelle. Revue Laryngol Otol Rhinol 1990 ; 111 :19-22. 14-Salvinelli F, Casale M, Di Peco V, Greco F, Trivelli M. Stapedoplasty in patients with small air-bone gap: why not? Med Hypotheses 2003; 60: 535-7.

15-Simoncelli C,Ricci G, Trabalzini F, Gullà M, Faralli M, Molini E. Stapes surgery : a review of 515 operations performed from 1988 to 2002. Mediterr J Otol 2005; $1: 14-9$
16-Ben Arab S, Besbes G, Hachicha S. L'otospongiose dans les populations du nord de la Tunisie: épidémiologie et étiologie. Ann Otolaryngol Chir Cervicofac. 2001; 118: 19-25.

17-Khalifa A, El-Guindy A, Erfan F. Stapedectomy for far-advanced otosclerosis. J Laryngol Otol 1998; 112: 158-60.

18-Vartiainen E. Sex differences in patients with hearing impairments caused by otosclerosis. Eur Arch Otorhinolaryngol 1999; 256: 431-3.

19-Causse JB. Subcophoses et otospongioses. Traitement médical et chirurgical. Ann Otolaryngol Chir Cervicofac $1990 ; 107: 411-7$.

20-Star B, Sen D, Karahatay S, Birkent H, Yetiser S. Effect of cochlear reserve on postoperative outcome in otosclerosis. Eur Arch Otorhinolaryngol 2007; 264: 489-93.

21-Zaki Z, Wiener V, Corré A, Ayache D. Surdités mixtes profondes par otospongiose. Rev Laryngol Otol Rhinol. $2006 ; 127: 127-30$.

22-Bordure $\mathrm{P}$, Robier $\mathrm{A}$, Malard $\mathrm{O}$. Chirurgie de la platine du stapès. In : Chirurgie otologique et oto-neurologique. Masson, Paris, 2005, pp : 119-36.

23-Roulleau P, Martin C, Martin H, et al. Les techniques actuelles. In : Roulleau P, Martin C, Bebear JP, et al. L'otospongiose-otosclérose. Paris, Arnette 1994, pp: 133-153.

24-Rondini-Gilli E, Grayelli AB, Boutin P, et al. Otospongiose : techniques chirurgicales et résultats. A propos de 150 cas. Ann Otolaryngol Chir Cervicofac $2002 ; 119: 227-33$

25-Esquivel CR, Mamicoglu B, Wiet RJ. Long-term results of small fenestra stapedectomy compared with large fenestra technique. Laryngoscope 2002; 112: 1338-41.

26-Ayache D, Sleiman J, Tchuente AN, Elbaz P. Variantes et incidents per-opératoires observés au cours de la chirurgie de l'otospongiose. Ann Otolaryngol Chir Cervicofac $1999 ; 116: 8-14$

27-Ballester M, Blaster B, Hausler R. Stapédotomie et variants anatomiques du nerf facial. Rev Laryngol Otol Rhinol $2000 ; 121: 181-6$.

28-Daniels RL, Krirger LW, Lippy WH. The other ear: findings and results in 1800 bilateral stapedectomies. Otol Neurotol 2001; 22: 603-7.

29-Couvreur P, Baltazar B, Lacher G, Fillippini JF, Vincey P. Geyser lors de la chirurgie de l'otospongiose. Rev Laryngol Otol Rhinol 2003 ; 124 : 31-7. 